\title{
Hypoxia-induced inflammation and purinergic signaling in cross clamping the human aorta
}

\author{
Juho Jalkanen ${ }^{1 *}$, Mikael Maksimow², Sirpa Jalkanen² and Harri Hakovirta ${ }^{1}$
}

\begin{abstract}
Open aortic surgery evokes a systemic inflammatory response and is associated with high morbidity and mortality. Purinergic signaling has been shown to be crucial for maintaining vascular integrity and attenuating inflammation related to hypoxia. The involvement of purinergic signaling in cross clamping of major human arteries is unknown. Our aim was to compare systemic inflammatory responses and hypoxia-induced purinergic signaling in patients undergoing either open infra-renal abdominal aortic repair or infra-inguinal revascularization. Pre- and $24 \mathrm{~h}$ postoperative blood samples were gathered from 6 patients undergoing aortic clamping and 6 similar patients undergoing common femoral artery cross-clamping. Using Biorad Multipex ${ }^{\mathrm{TM}} 21$ - and 27-panels 48 different cytokines, chemokines and growth factors were analyzed, in addition to circulating levels of ATP, ADP, CD39, CD73 and HIF-1a, and compared between the groups. Several inflammatory cytokines were elevated from baseline levels after aortic clamping, but not after femoral cross clamping. Most pronoun rises were seen in IL-6 (667 \%, $P=0.016)$ and HGF (760 \%, $P=0.016)$. HIF-1 a values showed a steady increase after clamping of either artery unless the subject underwent blood transfusion. Despite an adequate increase in HIF-1a CD39 and CD73 activity decreased significantly after aortic clamping ( $P=0.047$ and $P=0.016$, respectively). Aortic clamping is associated with a clear and strong systemic inflammatory response and impaired repair mechanisms in terms of purinergic signaling. Patients undergoing open aorta repair could benefit from pre-operative medical therapy, which enhances CD73 expression.
\end{abstract}

Keywords: Hypoxia, Aortic clamping, SIRS, IL-6, HGF, HIF-1a, CD73

\section{Background}

Acute organ injury remains one of the leading causes of morbidity and mortality in surgical patients (Eltzschig 2013). A highlight of such surgery is open aortic cross clamping and reconstruction, which is associated with a systemic inflammatory response syndrome (SIRS) (Bown et al. 2001; Vasdekis et al. 2008) and dysfunction of central organs, especially the lungs, kidneys and intestine (Brady et al. 2000; Papia et al. 2006; Moris et al. 2014). Thus, open aorta repair has been referred to as the ultimate stress test (Ali et al. 2008). Patients undergoing major vascular surgery are also often burdened by other significant cardiovascular

\footnotetext{
*Correspondence: juho.jalkanen@utu.fi

${ }^{1}$ Department of Vascular Surgery, Turku University and Turku University

Hospital, Hämeenkatu 11, 20521 Turku, Finland

Full list of author information is available at the end of the article
}

illnesses. This is especially the case amongst patients with peripheral artery disease (PAD), for whom the prevalence of cardiovascular co-morbidities is significantly elevated (Gallino et al. 2014). It has been shown that patients undergoing surgery of the lower limb for PAD have even more peri/post-operative myocardial morbidity than do patients undergoing aorta repair due to abdominal aortic aneurysm (AAA), but yet patients undergoing aortic surgery have a worse scenario (Krupski et al. 1992; Ali et al. 2008). This has lead to the conception that cross clamping of the infra-renal abdominal aorta leads to a markedly different hypoxia-induced pathophysiologic response than the crossclamping of the iliac or common femoral artery.

Extra cellular CD73 (ecto-5'-nucleotidase/NT5E) derived adenosine production is considered a key pathway in attenuating hypoxia-induced inflammation (Thompson et al. 2004; Eltzschig et al. 2012) and protective of several central

\section{望 Springer}


organs (Grenz et al. 2007; Eckle et al. 2007; Hart et al. 2011). The key driver of this response is hypoxia-induced factor-1 alpha (HIF-1 $\alpha$ ). However, the current background literature of this pathway is mostly derived from animal studies, in which cross-clamping of central arteries has been widely used to provoke reperfusion injury and a systemic inflammatory response in order to study different organ failures (Grenz et al. 2007; Kiss et al. 2007; Hart et al. 2011).

Thus, in this study our objective was to study the systemic inflammatory and growth factor response of aortic cross clamping. In addition, to further understand hypoxia-induced repair mechanisms in relation to cross clamping of a major artery, components of purinergic signaling were analyzed. Pre- and post-operative levels of 48 different cytokines, chemokines and growth-factors, in addition to ATP, ADP, CD39, CD73 and HIF-1 $\alpha$, were analyzed from blood samples of six PAD patients undergoing open repair of the infra-renal abdominal aorta and were compared to six similar risk factor matched PAD patients undergoing infra-inguinal vascular surgery and cross-clamping of the common femoral artery.

\section{Results and discussion}

Description of the study subjects

The study group baseline data is presented in Table 1. The groups were well matched and were mostly without any statistically significant differences. Patients undergoing open aorta repair tended to be a bit younger and had slightly less renal insufficiency and coronary artery disease (CAD), see Table $1 \mathrm{~A}$. The groups were also comparable based on major issues relating to procedural variables such clamping time and blood loss (Table 1B). The only significant difference was seen in the amount of transfusion of saline products received between the sampling. Subjects undergoing open aorta repair received significantly more crystalloid transfusion than did subjects undergoing infra-inguinal surgery $(P=0.03)$.

\section{Aortic clamping significantly elevated levels of several cytokines, especially IL-6 and HGF}

The majority of the values for IFN- $\alpha 2$, LIF, IL- $1 \alpha$, IL-3, IL-12p40, IL-15, bFGF, RANTES and TNF- $\beta$ were below the detection limit, and were left out of the statistical analysis.

For aortic clamping there was a significant rise in the level of IL-6 (667 \%, $P=0.016)$, IL-8 (75 \%, $P=0.047)$, IL-2R $\alpha(152 \%, P=0.031)$, IL-16 (129 \%, $P=0.031)$, GRO $\alpha(252 \%, P=0.031)$, HGF (760 \%, $P=0.016)$, M-CSF (853 \%, $P=0.016)$, and SDF- $1 \alpha$ (447 \%, $P=0.016)$ using matched pair values across time and the Wilcoxon signed rank test. For cross clamping of the common femoral artery there was only a significant

Table 1 Description of baseline characteristics between patients undergoing clamping of the aorta vs. common femoral artery

\begin{tabular}{|c|c|c|c|}
\hline & Aorta $(n=6)$ & Femoral $(n=6)$ & $P$ value \\
\hline \multicolumn{4}{|l|}{ A (Baseline characteristics) } \\
\hline Gender (F/M \%) & $50 / 50 \%$ & $33 / 67 \%$ & $N S^{*}$ \\
\hline Age (years) & $64.5(61-71)$ & $72.5(64-77)$ & $N S^{* *}$ \\
\hline Hypertension & $67 \%$ & $67 \%$ & $N S^{*}$ \\
\hline Coronary artery disease & $33 \%$ & $50 \%$ & $N S^{*}$ \\
\hline Dyslipidemia & $17 \%$ & $17 \%$ & $\mathrm{NS}^{*}$ \\
\hline COPD & $33 \%$ & $33 \%$ & $N S^{*}$ \\
\hline Diabetes & $17 \%$ & $17 \%$ & $N S^{*}$ \\
\hline Renal insufficiency & $17 \%$ & $33 \%$ & $N S^{*}$ \\
\hline Systolic BP (mmHg) & $160(146-174)$ & $148(133-152)$ & $N S^{* *}$ \\
\hline Creatine $(\mu \mathrm{mol} / \mathrm{L})$ & $78(61-101)$ & $92(68-136)$ & $N S^{* *}$ \\
\hline $\mathrm{CRP}(\mathrm{mg} / \mathrm{L})$ & $8(3-19)$ & $8(3-18)$ & $N S^{* *}$ \\
\hline \multicolumn{4}{|l|}{ B (Procedure related variables) } \\
\hline Procedure time (min) & $213(148-233)$ & $150(103-218)$ & $N S^{* *}$ \\
\hline Clamping time (min) & 75 (45-98) & $78(38-135)$ & $N S^{* *}$ \\
\hline Blood loss (mL) & $1025(500-2890)$ & $250(88-1425)$ & $N S^{* *}$ \\
\hline $\begin{array}{l}\text { Amount of saline transfusion between sampling } \\
(\mathrm{mL})\end{array}$ & $6250(4575-8800)$ & $2975(2575-5100)$ & $0.03^{* *}$ \\
\hline Time from clamping to second sample $(\mathrm{h})$ & $29(22-38)$ & $21(20-28)$ & $N S^{* *}$ \\
\hline
\end{tabular}

Prevalence presented as percentage and numerical values as median and inter-quartile range (IQR). Difference between groups calculated using the * Chi square test and ${ }^{* *}$ Mann-Whitney $U$ test 
increase in the level of IL-6 $(96 \%, P=0.031)$ and GRO $\alpha$ $(84 \%, P=0.031)$. All of the cytokines, which underwent a significant alteration due to clamping were then tested for the effect of aortic clamping versus femoral artery clamping between the groups. These results are presented in Table 2. First, the pre-operative baseline values between the groups were tested to ensure that the effect of clamping was not affected by any underlying baseline differences. No significant differences in the cytokine levels between the groups could be seen in preoperative sampling. For aortic clamping the most pronoun change was observed in the elevation of IL-6, when compared to femoral clamping (667 vs. $96 \%$, respectively, $P=0.006)$. As was also seen for HGF $(760 \%$ increase for aortic clamping vs. $33 \%$ increase for femoral clamping, $P=0.045)$. Figure 1 illustrates the differences of aortic vs. femoral clamping on IL-6 and HGF as matched pairs. All the corresponding raw values are presented in Additional file 1: Table S1. Other cytokines failed to show statistical increase in the aortic clamping when compared to the femoral clamping as matched pairs.

\section{CD39 and CD73 decrease significantly after clamping aortic clamping}

Baseline values of CD39 and CD73 did not differ between groups (Table 3). After aortic clamping both CD39 and CD73 significantly declined from baseline levels, see

Table 2 Baseline values of significantly altered cytokines presented as median and inter-quartile range (IQR) and average percentage of the change after cross clamping of the aorta vs. common femoral artery

\begin{tabular}{llll}
\hline & Aorta & Femoral & P value* \\
\hline IL-6 & & & \\
Baseline $(p g / m L)$ & $15.3(10.8-17.7)$ & $12.5(11.1-24.5)$ & NS \\
\% change & $667 \%$ & $96 \%$ & 0.006 \\
IL-8 & $40.0(33.2-51.3)$ & $29.5(27.0-39.1)$ & NS \\
& $75 \%$ & $17 \%$ & 0.361 \\
IL-2Ra & $89.7(77.4-160)$ & $65(39.6-174)$ & NS \\
& $152 \%$ & $7 \%$ & 0.068 \\
IL-16 & $142(81-172)$ & $154(46-194)$ & NS \\
& $129 \%$ & $20 \%$ & 0.068 \\
GROa & $30.9(20.8-52.5)$ & $58.0(34.7-77.9)$ & NS \\
& $252 \%$ & $84 \%$ & 0.144 \\
HGF & $681(599-865)$ & $688(560-1225)$ & NS \\
& $760 \%$ & $33 \%$ & 0.045 \\
M-CSF & $10.3(5.45-15.9)$ & $11.1(4.33-57.5)$ & NS \\
& $853 \%$ & $197 \%$ & 0.055 \\
SDF-1a & $69.6(32.0-130)$ & $75.4(41.3-77)$ & NS \\
& $447 \%$ & $10 \%$ & 0.154 \\
\hline
\end{tabular}

* Mann-Whitney U test
Fig. 2 and Table 3. CD39 declined an average of $43 \%$ $(\mathrm{P}=0.047)$, ranging from 2 to $63 \%$ decline. A similar $46 \%$ decline was seen in $\mathrm{CD} 73(\mathrm{P}=0.016)$, ranging from 21 to $55 \%$ decline (Fig. 2). In cross clamping of the common femoral artery there was no significant decrease in CD39, but a significant decrease in CD73 activity (Fig. 2). CD39 decreased an average of $6 \%$, ranging from $13 \%$ increase to $24 \%$ decrease. After common femoral artery clamping CD73 had a more constant, yet significant average $25 \%$ decrease, ranging from 16 to $30 \%$ decrease, $\mathrm{P}=0.016$ ). Thus, a stronger decline was seen in aortic clamping than in clamping of the femoral artery in both CD39 (43 vs $6 \%$ decrease, respectively, $\mathrm{P}=0.037$ ) and CD73 (46 vs. $25 \%$ decrease, respectively, $\mathrm{P}=0.11$ ), but for CD39 this was more evident. Circulating ATP and ADP levels showed no consistent or significant changes between pre- and post-operative samples in either procedure. Both increases and decreases were seen between pre- and post-operative levels, which amounted to slight but non-significant decrease in both ATP and ADP (Table 3). All raw values of components of purinergic signaling are presented in Additional file 1: Table S1.

\section{HIF-1a increased after clamping unless the subject underwent blood transfusion}

HIF- $1 \alpha$ was mostly associated with an increase after clamping in both groups. However, significant blood loss and consequent blood transfusion was associated with an opposing effect. All subjects who received blood transfusion had a decrease in HIF- $1 \alpha$ (average decrease $34 \%$, range $0-87 \%$ decrease). Subjects undergoing aortic clamping and not receiving blood transfusion had average $17 \%$ increase in HIF- $1 \alpha$ ranging from $15-61 \%$, and subject undergoing clamping of the femoral artery and not receiving blood transfusion had an average HIF- $1 \alpha$ increase of $18 \%$, ranging from 11-36\%. Although there was a tendency of higher HIF- $1 \alpha$ response after aortic clamping there was no significant difference between HIF- $1 \alpha$ in the groups. The HIF- $1 \alpha$ response between the pre- and post-operative values was however significantly affected depending on whether the blood transfusion was received or not $(\mathrm{P}=0.007)$, see Fig. 3 .

\section{Discussion of results}

This study clearly shows that open infra-renal aorta repair significantly elevates levels of several circulating cytokines and growth factors, but in particular IL- 6 and HGF. Both markers have been associated with worse disease prognosis in SIRS, sepsis, ARDS and severe acute pancreatitis (Parsons et al. 2005; Nieminen et al. 2014).

The role of IL- 6 as an acute phase pro-inflammatory mediator is unquestionable. However, the elevation of HGF relating to SIRS after open aorta repair is a novel 


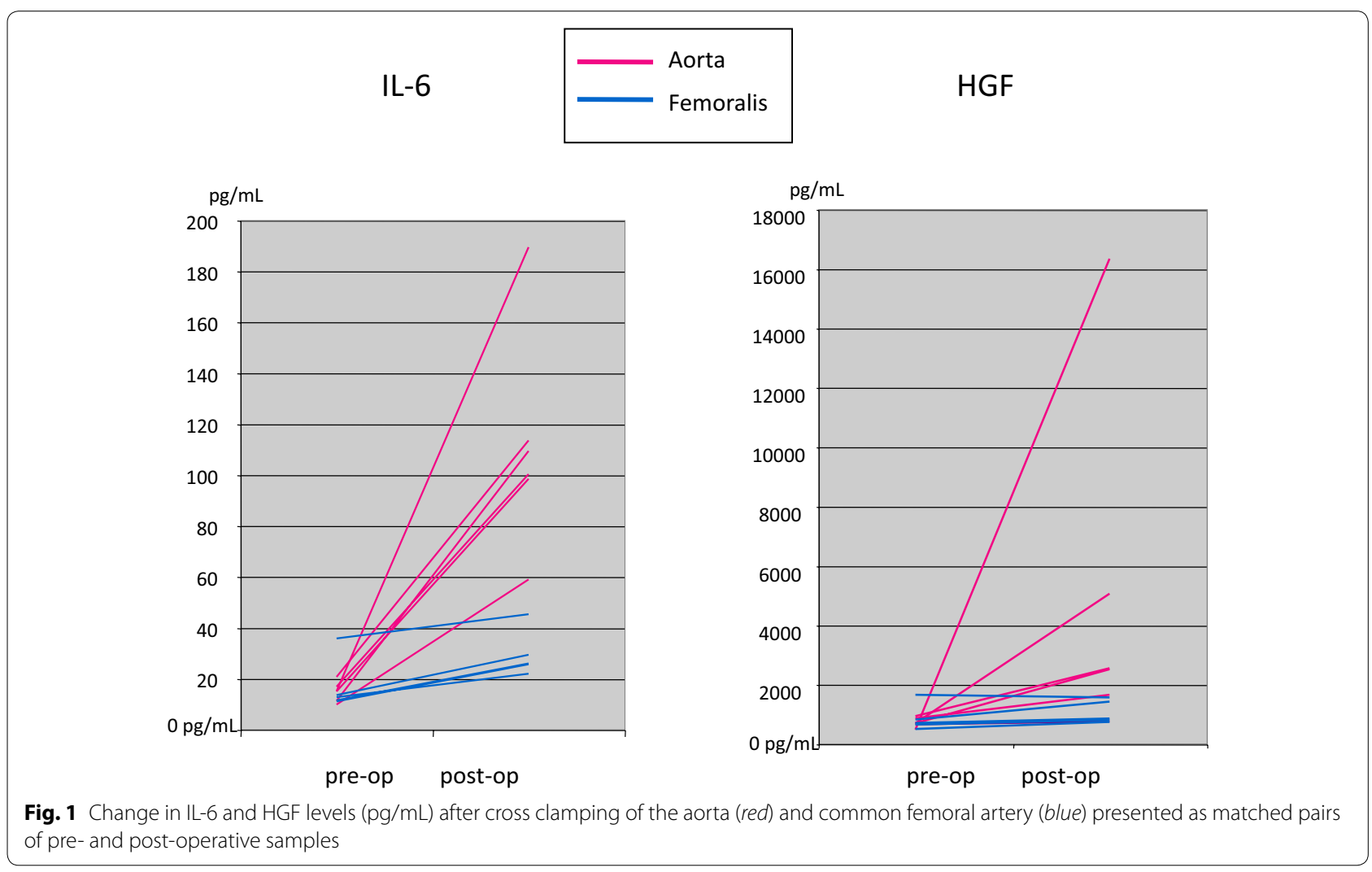

Table 3 Baseline values of ATP, ADP, CD39 and CD73 presented as median and range, and average percentage change (and range) after clamping

\begin{tabular}{|c|c|c|c|c|c|c|}
\hline & Aorta baseline & Average change & $P$ value $*$ & Femoral baseline & Average change & $P$ value* \\
\hline ATP (nmol/L) & $3276(1582-4476)$ & $-6 \%(-30$ to $50 \%)$ & NS & $4336(2731-5187)$ & $-21 \%(-42$ to $21 \%)$ & NS \\
\hline $\mathrm{ADP}(\mathrm{nmol} / \mathrm{L})$ & $2790(1243-3317)$ & $-9 \%(-45$ to $29 \%)$ & NS & 1755 (1219-3944) & $-4 \%(-56$ to $71 \%)$ & NS \\
\hline CD39 (nmol/mL/h) & $20.5(13-27)$ & $-43 \%(-2$ to $-63 \%)$ & 0.047 & $15(8-20)$ & $-6 \%(-24$ to $13 \%)$ & NS \\
\hline CD73 $(\mathrm{nmol} / \mathrm{mL} / \mathrm{h})$ & $243(184-251)$ & $-46 \%(-21$ to $-55 \%)$ & 0.016 & $303(224-549)$ & $-25 \%(-16$ to $-30 \%)$ & 0.016 \\
\hline
\end{tabular}

Significance of change after clamping calculated using matched pairs

* Wilcoxon sign rank test

finding. The role of HGF is controversial according to the background literature. HGF is considered anti-inflammatory by nature, and a necessary mediator of repair mechanisms (Shimizu et al. 2012). In several animal models it has been considered protective and even a possible target for drug development to prevent organ failure (Gong 2008). HGF has been shown to down regulate inflammation and leukocyte extravasation by attenuating TNF $\alpha$ induced expression of intercellular adhesion molecule-1 (ICAM-1), vascular adhesion molecule-1 (VCAM-1) (Mine et al. 1998), and E-selectin (Gong et al. 2006). However, this study indicates that the inflammatory response of open aorta repair is not TNF $\alpha$ mediated, as TNF $\alpha$ was not affected from cross clamping of the aorta. Thus, the HGF response in open aorta repair could be an acute repair mechanism in response to severe hypoxia. Despite of its known anti-inflammatory mechanisms, HGF has several pleiotropic actions, and has been shown to be a potent mediator of neutrophil adhesion via integrin activation (Mine et al. 1998) and is highly expressed in polymorphonuclear leukocytes at acute sites of inflammation (Matsushima et al. 2004). Neutrophil infiltration highlights sites of acute inflammation, especially in response to ischemia and reperfusion. This may explain why HGF is highly elevated in severe acute conditions and may be a predictor of poor prognosis. According to the results of this study HGF had a strong positive correlation with IL-6 $(R=0.52, P=0.01$, data not shown).

All this translates to the conclusion that it is namely the hypoxia induced systemic acute inflammatory response 


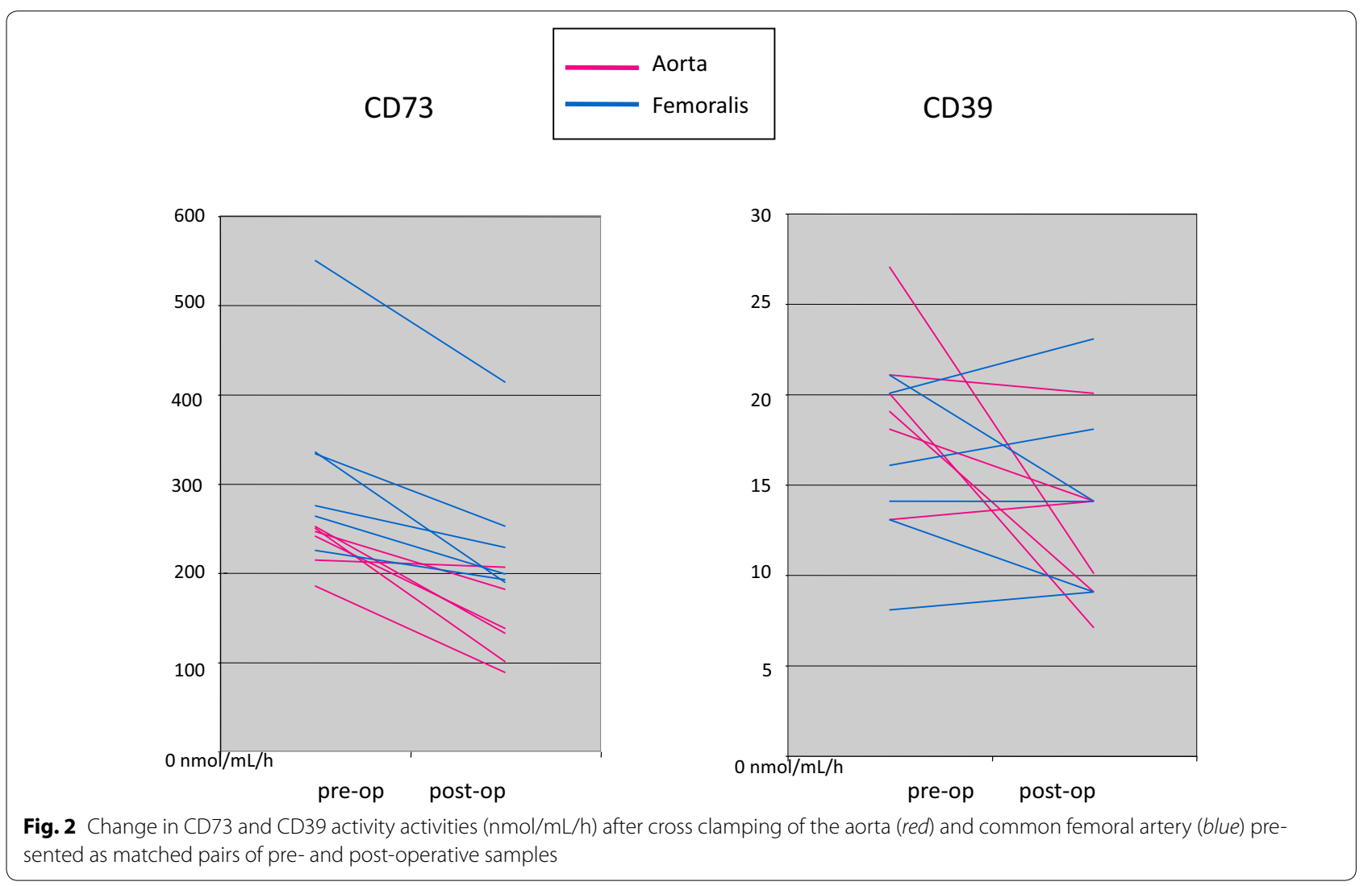

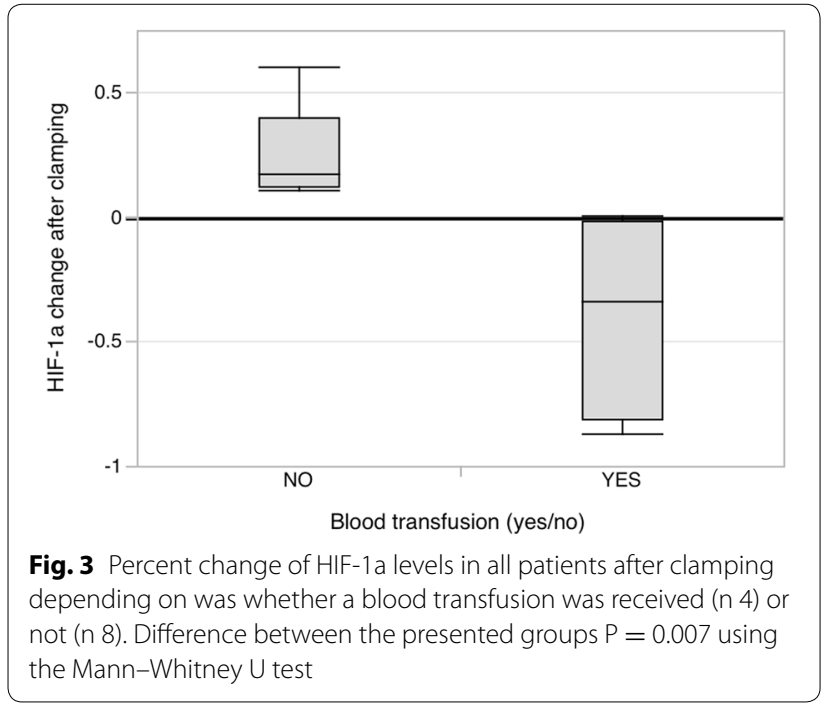

that makes aortic clamping the strain it is. One could argue that the elevation in circulating cytokines seen here is a response to the overall surgical trauma patients undergo in open repair, which is extensive. Most likely surgical trauma does contribute to some of the elevation, e.g. in more slowly acting cytokines (IL-8 and IL-16) and growth factors (GRO $\alpha$ and SDF-1 $\alpha$ ) as seen here, similar to the rise of CRP seen later on after a major surgery. However, here we have shown a dramatic acute proinflammatory response in the elevation of IL- 6 and HGF, which are known to be induced by hypoxia and central tissue resections, but not by surgical trauma per se (Yamada et al. 1998). The known excessive need for transfusion of crystalloid fluids relating to open aorta repair, as seen in this study (Table 1), would more likely have a diluting rather than a lifting affect on cytokine levels.

The excessive need for fluid transfusion to maintain an optimum hemodynamic level relating to clamping and un-clamping of the aorta can in part be a result of impaired adenosine production as seen here in decreased CD39 and CD73 activity especially in subjects undergoing aortic clamping. To some extent CD39 activity even increased in subjects undergoing femoral clamping (Fig. 2). HIF- $1 \alpha$ activated adenosine production by CD73 is considered the key of maintaining vascular integrity and preventing vascular leak (Thompson et al. 2004; Hart et al. 2011). For patients undergoing open aorta repair the essence of this mechanism is even more crucial due to the high volume of transfused fluids. But why do CD39 and CD73 levels significantly decline in subjects undergoing aortic clamping at the first post-operative day, 
when they should go up? Hypoxia is a known key driver of CD39 and CD73 activity (Semenza 2010; Schwartz et al. 2011). An adequate elevation is seen in HIF-1 $\alpha$, unless blood loss was significant and the subject underwent blood transfusion (Fig. 3). It is a novel finding that blood transfusion impairs the HIF-1 $\alpha$ response, but helps to explain why blood transfusion is significantly associated with worse outcome and ischemia related morbidity (Murphy et al. 2007; Marik and Corwin 2008; Patel et al. 2014). This, however, is inadequate to explain an impaired CD39/CD73 response since this was seen in all subjects, even in subjects without blood transfusion and with an adequate elevation of HIF- $1 \alpha$. Perhaps CD39 and CD73 were consumed to compensate for elevated ATP and ADP levels (Yegutkin 2015) as ATP and ADP levels showed no consistent or significant alterations in either direction between the pre- and post-operative samples. Or perhaps, despite an increased expression at the endothelium, CD39 and CD73 do not shed into circulation as anticipated. Thus, an increase is not yet seen in soluble values measured here at the first post-operative day. A similar late rise in soluble activity of CD73 is seen when its expression is induced with the administration of intra venous interferon beta in humans (Bellingan et al. 2014).

\section{Limitations of the study}

The current study has marked limitations. First, and most importantly, this study is highly limited by the low number of study subjects. Due to the low number of subjects it is virtually impossible to find statistical differences between the baseline characteristics of the study groups. Despite this dilemma, the differences between the observed inflammatory responses and repair mechanisms, on the other hand, are statistically strong and quite clear. Thus, we postulate that the observed molecular changes would only become stronger with a bigger study population. Our objective was to capture true PAD patients undergoing aortic clamping and study the observed mechanisms. These patients were scarce. During the study period several open AAA procedures were performed. These patients were not included, because the baseline values of cytokines and purinergic signaling can be significantly altered due to PAD (Jalkanen et al. 2015). An additional limitation is that we only focused on two distinct time points: the morning prior to the operation and the morning of the first post-operative day. However, there is a clear rationale for this. IL-6 has been shown to be a key measure of SIRS and predict poor outcome in several conditions (Sakon et al. 1996; Matsushima et al. 2004; Nieminen et al. 2014), including major surgery (Yamada et al. 1998; Namekata et al. 2000). IL-6 levels peek at the first post-operative day, at around $24 \mathrm{~h}$, while for example CRP rises later on after a major surgery (Vasdekis et al. 2008; Watt et al. 2015). Similarly, organ failures commonly start to develop during the first post-operative day and become evident at the second, or even on the third post-operative day. Thus, we postulate that the first post-operative day is a watershed and as IL-6 peeks then, the repair mechanisms should also be in action at this time point. From the studies reporting increased CD73 expression in humans (Bellingan et al. 2014) we can also postulate that after HIF- $1 \alpha$ activation CD73 expression should peek at the first post-operative day.

\section{Conclusions}

Open infra-renal aorta repair is associated with a systemic inflammatory response, but in addition according to the present study an impaired CD39 and CD73 response to attenuate inflammation. This helps to explain severe morbidity and mortality related to open aorta repair. In conclusion, we wish to point out that despite a small study the witnessed phenomenon is obvious and merely proposes an explanatory molecular mechanism behind the burden of aortic clamping. Hypoxia induced inflammation and impaired hypoxia related repair mechanisms seen here relating to open aorta repair require further research and could serve as a target for operative preconditioning and better patient outcome. Such a therapy could be pre-operative statin loading, which is in common use for example in coronary artery bypass grafting (CABG). Statins are known for their anti-inflammatory properties and have been shown to have beneficial effects on CD39 and CD73 levels (Kaneider et al. 2002; Jalkanen et al. 2015). Pre-operative statin therapy has also been associated with better outcome after major vascular surgery (Durazzo et al. 2004; Le Manach et al. 2011; Sanders et al. 2013). However, we still lack a decisive multicenter randomized trial on the effect statin loading on infra-renal aorta repair against SIRS and organ dysfunction. Another possible therapy could be preoperative administration of intravenous interferon beta. It is known to up-regulate CD73, attenuate inflammation and prevent vascular leakage especially in the lungs (Kiss et al. 2007), and has shown promising initial results in the treatment of ARDS in humans (Bellingan et al. 2014).

\section{Methods}

\section{Study subjects}

As a part of the PURE ASO Study (The Role of Purinergic Signaling in Atherosclerosis, approved by the local Ethical Committee of the Hospital District of Southwestern Finland) consecutive blood samples were gathered for 1 year from non-urgent elective PAD patients admitted to the Department of Vascular Surgery of Turku University 
Hospital, Finland (Jalkanen et al. 2015). The department is a primary vascular surgery referral center for a population of 360,000 inhabitants. During enrollment 227 suitable patients were seen and 226 gave written informed consent. Out of these 226 PAD patients 6 went through open infra-renal abdominal aorta repair ( 1 for Leriche's Syndrome, and 5 for TASC D aorta-iliac lesions accompanied with a repairable AAA). For these 6 subjects undergoing aortic clamping 6 age and risk factor matched subjects undergoing common femoral artery clamping during the same hospital stay as the aortic reconstructions were randomly picked as control subjects (5 of these subjects had femoral endarcterectomy done and 1 subject underwent a femoropopliteal bypass to the proximal popliteal artery with a vascular prostheses). All 12 study subjects had claudication as their manifestation of PAD. For these 12 study subjects both pre- and postoperative blood samples were drawn. Otherwise, the PURE ASO Study population only went through the preoperative blood sampling.

\section{Blood sampling and analyses}

All samples were serum from centrifuged whole blood samples and plasma EDTA. The first samples were drawn in the morning of the operative day after over night fasting. The second sample was drawn the following morning after the operation. At this point patients were still fasting. After centrifugation the serum samples were stored at $-70{ }^{\circ} \mathrm{C}$ until analyses. All analyses were done at once with the same magnetic bead suspension array kit of Bio-Plex Pro Human Cytokine 21- and 27-plex panels (Bio-Rad, Hercules, CA, USA) according to the manufacturer's instructions except that the amount of beads, detection antibodies and streptavidin-phycoerythrin conjugate were used at half of their recommended concentration as described previously (Nieminen et al. 2014). The results were analyzed using the Bio-Plex 200 System, and calculated using the Bio-Plex Manager 6.0 software (Bio-Rad Laboratories, Hercules, CA, USA). The persons doing the cytokine analysis were unaware of patient procedures.

The 21-plex panel included interleukin $1 \alpha$ (IL-1 $\alpha)$, IL-2 receptor $\alpha(\mathrm{IL}-2 \mathrm{R} \alpha)$, IL-3, IL-12p40, IL-16, IL-18, cutaneous $\mathrm{T}$ cell attracting chemokine (CTACK), growthregulated oncogene $\alpha$ (GRO $\alpha)$, hepatocyte growth factor (HGF), interferon $\alpha 2$ (IFN- $\alpha 2$ ), leukemia inhibitory factor (LIF), monocyte chemotactic protein 3 (MCP-3), macrophage colony-stimulating factor (M-CSF), macrophage migration inhibitory factor (MIG), monokine induced by IFN- $\gamma$ (MIF), $\beta$-nerve growth factor ( $\beta$-NGF), stem cell factor (SCF), stem cell growth factor $\beta$ (SCGF- $\beta$ ), stromal cell-derived factor $1 \alpha$ (SDF-1 $\alpha)$, tumor necrosis factor $\beta$ (TNF- $\beta$ ) and TNF-related apoptosis inducing ligand
(TRAIL). The 27-plex included IL-1 $\beta$, IL-1 receptor antagonist, IL-2, IL-4, IL-5, IL-6, IL-7, IL-8, IL-9, IL-10, IL-12p70, IL- 13, IL-15, IL-17, basic fibroblast growth factor (bFGF), eotaxin, granulocyte colony-stimulating factor (G-CSF), granulocyte- macrophage colony-stimulating factor (GM-CSF), IFN- $\gamma$, IFN- $\gamma$-induced protein 10 , monocyte chemotactic protein 1 (MCP-1), macrophage inflammatory protein $1 \alpha$ (MIP- $1 \alpha)$, MIP- $1 \beta$, platelet-derived growth factor (PDGF), regulated on activation normal $\mathrm{T}$ cell expressed and secreted (RANTES), TNF- $\alpha$ and vascular endothelial growth factor (VEGF).

\section{Measurement of soluble nucleotidase (CD39 and CD73) activities in human serum}

For ADPase/NTPDase activity, serum $(10 \mu \mathrm{L})$ was incubated for $60 \mathrm{~min}$ at $37^{\circ} \mathrm{C}$ in $80 \mu \mathrm{L}$ of RPMI-1640 medium containing $5 \mathrm{mM} \beta$-glycerophosphate, $80 \mu \mathrm{M}$ adenylate kinase inhibitor $\mathrm{Ap}_{5} \mathrm{~A}$, and $50 \mu \mathrm{M}$ ADP with a $\left[2,8-{ }^{3} \mathrm{H}\right]$ ADP tracer (Perkin Elmer, Boston, USA). Likewise, $5^{\prime}$-nucleotidase activity was assayed by incubating $10 \mu \mathrm{L}$ of serum for $60 \mathrm{~min}$ with $300 \mu \mathrm{M}\left[2-{ }^{3} \mathrm{H}\right] \mathrm{AMP}$ (Quotient Bioresearch, GE Healthcare, Rushden, UK). Radiolabelled substrates and their dephosphorylated products were separated by thin-layer chromatography and quantified by scintillation $\beta$-counting. Enzymatic activities were expressed as nanomoles of ${ }^{3} \mathrm{H}$-substrate metabolized per hr by $1 \mathrm{~mL}$ of serum (Yegutkin 2015).

\section{Quantification of ATP and ADP levels in human plasma}

Briefly as described previously (Yegutkin et al. 2003), 10 $\mu \mathrm{L}$ aliquots of EDTA plasma were transferred into two parallel wells of a white non-phosphorescent 96-well microplate containing $100 \mu \mathrm{L}$ of PBS with (A) or without (B) a solution of $200 \mu \mathrm{M}$ UTP and $5 \mathrm{U} / \mathrm{mL}$ NDP kinase from baker's yeast $S$. cerevisiae (Sigma). Following the addition of $50 \mu \mathrm{L}$ of ATP-monitoring reagent, sample luminescence was measured using a Tecan Infinite M200 microplate reader (Salzburg, Austria). The difference in luminescence signals between well "A" (ATP + ADP) and "B" (only ATP) enabled the quantification of ADP concentration, which was converted into ATP through an NDP kinase-mediated reaction in the presence of exogenous UTP. This approach allows simultaneous measurement of both ATP and ADP content within the same sample.

\section{Measurement of HIF-1 a Activity in Human serum}

HIF-1a activity of serum samples was analyzed using ELISA kit of Elabscience (Wuhan, China) according to the manufacturer's instructions. The optical density (OD) values were read using Tecan Infinite M200 and Magellan 7.2 software for Microsoft Windows (Tecan Group, Männedorf, Switzerland). 


\section{Statistical analysis}

Statistical analyses were performed using JMP 11.1 Pro statistical software from SAS (SAS Institute Inc., Cary, NC, USA). Baseline characteristics of subjects are reported using medians and inter-quartile range (IQR). Comorbidities were gathered on a yes/no basis and are presented as a percentage of prevalence amongst subjects. Difference between prevalence was compared using the Chi square test, and difference between numeric values between the groups using the Mann-Whitney $\mathrm{U}$ test. Cytokine results are given as median and IQR. Changes in cytokine levels after clamping are presented as percentage rise or fall from the baseline value. The significance of the change of each cytokine level and component of purinergic signaling in relation to clamping was measured using matched pair values across time and the Wilcoxon signed rank test. Cytokine levels that changed significantly because of clamping were then compared between the two study groups (aorta vs. common femoral artery) using the Mann-Whitney U test.

\section{Additional file}

Additional file: Table S1. Raw data on patient baseline characteristics, procedural variables, and essential pre- and post-operative analyses.

\section{Authors' contributions}

Initial idea and study design by JJ. $\mathrm{HH}$ and $\mathrm{JJ}$ collected the data. MM, JJ and SJ contributed to the analysis of data. All authors read and approved the final manuscript.

\section{Author details \\ ${ }^{1}$ Department of Vascular Surgery, Turku University and Turku University Hospital, Hämeenkatu 11, 20521 Turku, Finland. ${ }^{2}$ MediCity Research Labora- tory, Department of Microbiology and Immunology, University of Turku, Tykistönkatu 6A, 20520 Turku, Finland.}

\section{Acknowledgements}

From the Medicity Research Laboratory of Turku University (Turku, Finland) we thank Sari Mäki and Teija Kanasuo for technical assistance and Ph.D. Gennady G. Yegutkin for analyses of components of purinergic signaling and critical review of the manuscript.

\section{Competing interests}

Juho Jalkanen, Mikael Maksimow and Sirpa Jalkanen own stock of Faron Pharmaceuticals Ltd., Turku, Finland, which develops IFN-beta treatment for organ injury.

\section{Funding}

The study was supported by the Academy of Finland, the Sigrid Juselius Foundation, the Aarne Koskelo Foundation, and the Clinical Research Fund (EVO) of Turku University Hospital.

Received: 5 November 2015 Accepted: 21 December 2015

Published online: 04 January 2016

\section{References}

Ali ZA, Callaghan CJ, Ali AA et al (2008) Perioperative myocardial injury after elective open abdominal aortic aneurysm repair predicts outcome. Eur J Vasc Endovasc Surg 35:413-419. doi:10.1016/j.ejvs.2007.10.007
Bellingan G, Maksimow M, Howell DC et al (2014) The effect of intravenous interferon-beta-1a (FP-1201) on lung CD73 expression and on acute respiratory distress syndrome mortality: an open-label study. The Lancet Respiratory Medicine 2:98-107. doi:10.1016/ S2213-2600(13)70259-5

Bown MJ, Nicholson ML, Bell PR, Sayers RD (2001) Cytokines and inflammatory pathways in the pathogenesis of multiple organ failure following abdominal aortic aneurysm repair. Eur J Vasc Endovasc Surg 22:485-495. doi:10.1053/ejvs.2001.1522

Brady AR, Fowkes FG, Greenhalgh RM et al (2000) Risk factors for postoperative death following elective surgical repair of abdominal aortic aneurysm: results from the UK Small Aneurysm Trial. On behalf of the UK Small Aneurysm Trial participants. Br J Surg 87:742-749. doi:10.1046/j.1365-2168.2000.01410.x

Durazzo AES, Machado FS, Ikeoka DT et al (2004) Reduction in cardiovascular events after vascular surgery with atorvastatin: a randomized trial. J Vasc Surg 39:967-975. doi:10.1016/j.jvs.2004.01.004 (discussion 975-6)

Eckle T, Füllbier L, Wehrmann M et al (2007) Identification of ectonucleotidases CD39 and CD73 in innate protection during acute lung injury. J Immunol 178:8127-8137

Eltzschig HK (2013) Targeting purinergic signaling for perioperative organ protection. Anesthesiology 118:1001-1004. doi:10.1097/ ALN.0b013e3182874686

Eltzschig HK, Sitkovsky MV, Robson SC (2012) Purinergic signaling during inflammation. N Engl J Med 367:2322-2333. doi:10.1056/NEJMra1205750

Gallino A, Aboyans V, Diehm C et al (2014) Non-coronary atherosclerosis. Eur Heart J 35:1112-1119. doi:10.1093/eurheartj/ehu071

Gong R (2008) Multi-target anti-inflammatory action of hepatocyte growth factor. Curr Opin Investig Drugs 9:1163-1170

Gong R, Rifai A, Dworkin LD (2006) Hepatocyte growth factor suppresses acute renal inflammation by inhibition of endothelial E-selectin. Kidney Int 69:1166-1174. doi:10.1038/sj.ki.5000246

Grenz A, Zhang H, Eckle T et al (2007) Protective role of ecto-5'-nucleotidase (CD73) in renal ischemia. J Am Soc Nephrol 18:833-845. doi:10.1681/ ASN.2006101141

Hart ML, Grenz A, Gorzolla IC et al (2011) Hypoxia-inducible factor-1adependent protection from intestinal ischemia/reperfusion injury involves ecto-5'-nucleotidase (CD73) and the A2B adenosine receptor. J Immunol 186:4367-4374. doi:10.4049/jimmunol.0903617

Jalkanen J, Yegutkin GG, Hollmén M et al (2015) Aberrant circulating levels of purinergic signaling markers are associated with several key aspects of peripheral atherosclerosis and thrombosis. Circ Res 116:1206-1215. doi:10.1161/CIRCRESAHA.116.305715

Kaneider NC, Egger P, Dunzendorfer S et al (2002) Reversal of thrombininduced deactivation of CD39/ATPDase in endothelial cells by HMG-CoA reductase inhibition: effects on Rho-GTPase and adenosine nucleotide metabolism. Arterioscler Thromb Vasc Biol 22:894-900

Kiss J, Yegutkin GG, Koskinen K et al (2007) IFN-beta protects from vascular leakage via up-regulation of CD73. Eur J Immunol 37:3334-3338. doi:10.1002/eji.200737793

Krupski WC, Layug EL, Reilly LM et al (1992) Comparison of cardiac morbidity between aortic and infrainguinal operations. Study of Perioperative Ischemia (SPI) Research Group. J Vasc Surg 15:354-363 (discussion 364-5)

Le Manach Y, Ibanez Esteves C, Bertrand M et al (2011) Impact of preoperative statin therapy on adverse postoperative outcomes in patients undergoing vascular surgery. Anesthesiology 114:98-104. doi:10.1097/ ALN.0b013e31820254a6

Marik PE, Corwin HL (2008) Acute lung injury following blood transfusion: expanding the definition. Crit Care Med 36:3080-3084. doi:10.1097/ cCM.0b013e31818c3801

Matsushima A, Ogura H, Koh T et al (2004) Hepatocyte growth factor in polymorphonuclear leukocytes is increased in patients with systemic inflammatory response syndrome. J Trauma 56:259-264. doi:10.1097/01. TA.0000111752.60500.DA

Mine S, Tanaka Y, Suematu M et al (1998) Hepatocyte growth factor is a potent trigger of neutrophil adhesion through rapid activation of lymphocyte function-associated antigen-1. Lab Invest 78:1395-1404

Moris DN, Kontos MI, Mantonakis El et al (2014) Concept of the aortic aneurysm repair-related surgical stress: a review of the literature. Int J Clin Exp Med 7:2402-2412 
Murphy GJ, Reeves BC, Rogers CA et al (2007) Increased mortality, postoperative morbidity, and cost after red blood cell transfusion in patients having cardiac surgery. Circulation 116:2544-2552. doi:10.1161/ CIRCULATIONAHA.107.698977

Namekata K, Takamori S, Kojima K et al (2000) Significant changes in the serum levels of IL-6, h-HGF, and type IV collagen 7S during the perioperative period of a hepatectomy: relevance to SIRS. Surg Today 30:403-409

Nieminen A, Maksimow M, Mentula P et al (2014) Circulating cytokines in predicting development of severe acute pancreatitis. Crit Care 18:R104. doi:10.1186/cc13885

Papia G, Klein D, Lindsay TF (2006) Intensive care of the patient following open abdominal aortic surgery. Curr Opin Crit Care 12:340-345. doi:10.1097/01. ccx.0000235212.86859.54

Parsons PE, Eisner MD, Thompson BT et al (2005) Lower tidal volume ventilation and plasma cytokine markers of inflammation in patients with acute lung injury. Crit Care Med 33:1-6 (discussion 230-2)

Patel SV, Kidane B, Klingel M, Parry N (2014) Risks associated with red blood cell transfusion in the trauma population, a meta-analysis. Injury 45:15221533. doi:10.1016/j.injury.2014.05.015

Sakon M, Kita Y, Yoshida T et al (1996) Plasma hepatocyte growth factor levels are increased in systemic inflammatory response syndrome. Surg Today 26:236-241

Sanders RD, Nicholson A, Lewis SR et al (2013) Perioperative statin therapy for improving outcomes during and after noncardiac vascular surgery. Cochrane Database Syst Rev 7:CD009971. doi:10.1002/14651858. CD009971.pub2

Schwartz RS, Eltzschig HK, Carmeliet P (2011) Hypoxia and inflammation. N Engl J Med 364:656-665. doi:10.1056/NEJMra0910283
Semenza GL (2010) Vascular responses to hypoxia and ischemia. Arterioscler Thromb Vasc Biol 30:648-652. doi:10.1161/ATVBAHA.108.181644

Shimizu K, Taniyama Y, Sanada F et al (2012) Hepatocyte growth factor inhibits lipopolysaccharide-induced oxidative stress via epithelial growth factor receptor degradation. Arterioscler Thromb Vasc Biol 32:2687-2693. doi:10.1161/ATVBAHA.112.300041

Thompson LF, Eltzschig HK, Ibla JC et al (2004) Crucial role for ecto-5'nucleotidase (CD73) in vascular leakage during hypoxia. J Exp Med 200:1395-1405. doi:10.1084/jem.20040915

Vasdekis SN, Argentou M, Kakisis JD et al (2008) A global assessment of the inflammatory response elicited upon open abdominal aortic aneurysm repair. Vasc Endovascular Surg 42:47-53. doi:10.1177/1538574407308942

Watt DG, Horgan PG, McMillan DC (2015) Routine clinical markers of the magnitude of the systemic inflammatory response after elective operation: a systematic review. Surgery 157:362-380. doi:10.1016/j.surg.2014.09.009

Yamada T, Hisanaga M, Nakajima Y et al (1998) Serum interleukin-6, interleukin-8, hepatocyte growth factor, and nitric oxide changes during thoracic surgery. World J Surg 22:783-790

Yegutkin GG (2015) Enzymes involved in metabolism of extracellular nucleotides and nucleosides: functional implications and measurement of activities. Crit Rev Biochem Mol Biol 49:473-497. doi:10.3109/10409238. 2014.953627

Yegutkin GG, Samburski SS, Jalkanen S (2003) Soluble purine-converting enzymes circulate in human blood and regulate extracellular ATP level via counteracting pyrophosphatase and phosphotransfer reactions. FASEB J 17:1328-1330. doi:10.1096/fj.02-1136fje

\section{Submit your manuscript to a SpringerOpen ${ }^{\circ}$ journal and benefit from:}

- Convenient online submission

- Rigorous peer review

- Immediate publication on acceptance

- Open access: articles freely available online

- High visibility within the field

- Retaining the copyright to your article

Submit your next manuscript at $>$ springeropen.com 